\title{
Florian Vogt, Die »Anleitung zur musikalischen Setzkunst« von Gottfried Heinrich Stölzel (1690-1749). Edition und Kommentar, Neu- münster: von Bockel 2018
}

Schlagworte/Keywords: counterpoint; Gottfried Heinrich Stölzel; historical models of composition; historische Satzlehre; Johann Joseph Fux; Kontrapunkt; trias harmonica

Die zunehmende Erschließung barocker Traktate durch Übersetzungen, Kommentare und Einordnung trägt maßgeblich zu einem tieferen Verständnis der musiktheoretischen Strömungen im 17. und 18. Jahrhundert bei. ${ }^{1}$ Durch das Aufzeigen von Entwicklungslinien musiktheoretischen Denkens und ihren Querverbindungen werden Quellen nicht nur zugänglich, sondern auch in ihren Auswirkungen verständlich gemacht. Florian Vogt fügt den bisherigen Schriften mit Stölzels Anleitung zur musikalischen Setzkunst eine Quelle aus der Umbruchszeit des frühen 18. Jahrhunderts hinzu, deren Entstehung ca. 1735-38 zeitlich benachbart ist zu Rameaus Traité (1722) und Génération harmonique (1732), Fux' Gradus ad Parnassum (1725) - auf den sich Stölzel eng bezieht -, Heinichens Der Generalbaß in der Composition (1728) und Sorges Vorgemach der musicalischen Composition (1745). ${ }^{3}$

Schon Vogts Vortrag zu Stölzels FuxRezeption beim Essener Kongress der GMTH $2012^{4}$ machte das Potenzial einer Erschließung der Stölzel'schen Anleitung deutlich: »[Bei Stölzel] überlagern sich zwei Denkweisen: [Er] übernimmt und akzeptiert zum einen das sensualistische Erklärungsmodell, welches allgemein bewährte und geschätzte Klangverbindungen als srichtig، einschätzt; zugleich denkt er es aber mit den Kategorien des Kontrapunkts und der trias harmonica zusammen. ${ }^{5}$ Die Untersuchung von Stölzels musiktheoretischem Denken in der Anleitung und dessen Verortung in den zugehörigen Strömungen machen den Kern von Vogts Fragestellung aus; daran anschließend wird die Berechtigung des tradierten, eher abschätzigen Urteils ${ }^{6}$ über Stölzels von den Zeitgenossen durchaus geschätzte musiktheoretische Werke diskutiert (vgl. 25ff.). Der zweite Punkt ist zwar schnell zugunsten von Stölzel geklärt, aber insofern von Belang, als er beispielhaft ist für die durch die Fokussierung auf Rameau bedingte und bis vor Kurzem andauernde Vernachlässigung der deutschen Trias-harmonica-Lehre des 17 . Jahrhunderts als

1 Aus den letzten Jahren wären hier $u$. a. folgende Schriften zu nennen: Nathalie Meidhof (2016), Alexandre Étienne Chorons Akkordlehre. Konzepte, Quellen, Verbreitung, Hildesheim: Olms; Holtmeier 2017; Stephan Zirwes (2018), Von Ton zu Ton. Die Ausweichung in den musiktheoretischen Schriften des 18. Jahrhunderts, Kassel: Bärenreiter; Heffter i.V.; außerdem die äußerst hilfreiche Erschließung zahlreicher barocker Quellen in Ullrich Scheideler und Felix Wörner (Hg.) (2017), Musiktheorie von der Antike bis zur Gegenwart, Lexikon Schriften über Musik Bd. 1, Kassel: Bärenreiter.

2 Vgl. Vogts Datierung der Quelle, 39ff.

3 Zum Einfluss Stölzels auf Sorge - und damit auf die deutsche Musiktheorie des 18. Jahrhunderts insgesamt - vgl. Holtmeier 2017, 194-199.

6 Stölzel hat nach der Anleitung für die Mizler'sche Societät eine umfangreiche »[Abhandlung vom Recitativ]" geschrieben. Sie muss nach seinem Beitritt zur Societät 1739 entstanden sein, wurde allerdings nach seinem Tod nicht - wie ursprünglich vorgesehen - von Mizler veröffentlicht (vgl. Steger 1962, 23ff.). Lange Zeit wurde hauptsächlich dieser Rezitativtraktat wahrgenommen, daneben allenfalls noch der Kanontraktat (Practischer Beweiß, 1725); Stölzels andere Schriften wurden als uneigenständig und epigonenhaft beschrieben: eine auffällige Diskrepanz zwischen der hohen Wertschätzung für die Rezitativlehre und der Geringachtung der anderen Texte (ebd., 22f). 
der eigentlichen historischen Quelle des modernen stufentheoretischen Systems. ${ }^{7}$

Während Johannes Lippius' Idee der strias harmonica $\iota^{8}$ von vielen deutschen Theoretikern hauptsächlich als theoretisches Konzept aufgefasst wird und keinen Eingang in ihre praktischen Lehren findet, ${ }^{9}$ ist sie bei Wolfgang Caspar Printz ${ }^{10}$ bereits Erklärungsmodell der meisten musiktheoretischen Phänomene, wobei er eher die ausübenden Musiker als eine theoretische Kontemplation im Blick hat. ${ }^{11}$ Stölzel, als Komponist für seine kontrapunktische Fertigkeit, Vielseitigkeit und Innovation geschätzt, denkt die Trias-harmonica-Lehre weiter und verknüpft sie mit praktischen satztechnischen Unterweisungen. Umkehrungsdenken und Terzschichtung sind bereits in seinem Practischen Beweiß ${ }^{12}$ von 1725 Grundlage des Dreiklangsverständnisses, und der verminderte und der übermäßige Dreiklang werden als

7 Eine umfassende Perspektiven-Korrektur in diesem Zusammenhang hat Ludwig Holtmeier mit seiner Dissertation vorgenommen (Holtmeier 2017).

8 In Lippius' Synopsis Musicae Novae (1612) ist die strias harmonicas Ausgangspunkt seines ganzen Systems und gleichzeitig musikalische Erscheinungsform der göttlichen Trinität (Synopsis, f. F4). Sprick betont, dass durch seine Lehre von der strias harmonica » $[$.... Lippius und nicht etwa Zarlino oder Rameau - zum Begründer der modernen Akkordlehre wurde" (Sprick 2009, 376).

9 Heinichen geht - mit im Grunde sensualistischen Erklärungsmustern - allein vom Generalbass und der Oktavregel aus, die mathematische Trias-harmonica-Tradition wird gar nicht, Kontrapunkt kaum integriert. Fux wiederum gibt »als Theoretiker keinerlei Hinweise [...], wie sich [...] modernes harmonisches Denken in Dur- und Molltonarten mit den alten Systemen des stile antico sowie der trias harmonica vereinbaren bzw. zusammendenken lässt« (Vogt 2015, 116).

10 Phrynis Mitilenæus, Dresden 1696.

11 Vgl. Sprick 2009, 390f.

12 Der Practische Beweiß legt im Wesentlichen dar, wie durch Umkehrung, metrische Verschiebung, Änderung von Einsatzreihenfolge, Stimmanordnung etc. aus einem vierstimmigen >Canon perpetuus in der Quinte und Oktave zahlreiche weitere gewonnen werden können; die Dreiklangslehre wird hier nicht weiter ausgeführt. strias deficiens` bzw. ssuperfluas definiert und als eigenständige Dreiklänge integriert. ${ }^{13}$ Seine Anleitung bettet diese modernen Phänomene in die Traditionen des praktischen sstile anticosKontrapunkts und der theoretischen strias harmonica ein. ${ }^{14}$ Dabei treten in Stölzels skalenbasierter Dreiklangsordnung die Kategorien von Konsonanz und Dissonanz hinter die Einbettung in die strias` zurück:

Diese Dinge scheinen zwar Neuigkeiten zu seyn; allein in der That verhält es sich also, wie wir hernach sehen werden, und erscheinet hieraus, nebst der Unersättlichkeit des menschlichen Gehörs, auch der große Eindruck, welchen die $5^{\text {te }}$ perfecta und $3^{\text {tie }}$ major, als solche Consonanzen, die, wie wir oben gehört haben, propriæ und per se aus der Zertheilung der Octave entspringen, indemselben machen, also daß fast alles was nur den Namen von der $5^{\text {te }}$ und $3^{\text {tie }}$ führet, ihre Stelle in Concentu vertreten, und zum Grunde vieler, sonderlich heut zu Tage gebräuchlichen Harmonien dienen muß. (247)

Stölzels Traktat scheint konkret als Lehrbuch konzipiert, wobei allerdings weniger bestimmte satztechnische oder stilistische Anleitungen im Fokus stehen, als vielmehr die Entwicklung eines analytischen Blicks und eines kritischen Verständnisses des Tonsystems, wodurch der Komponist imstande sein soll, »[...] auf schöpferische Art und Weise und nichtsdestotrotz traditionsbewusst Neues zu erfinden « (177). So zeichnet den Traktat eine enge Verzahnung von hoch differenzierter Musik- und Kompositionspraxis und musiktheoretischer Reflexion in pythagoreischer Tradition aus. Gleichzeitig stellt Stölzel damit alte katholische Kontrapunktpraxis und neuere, die modernen DurMoll-Tonarten integrierende und mit harmonischer Theoriebildung eng verknüpfte protestantische Kantionalsatzpraxis einander gegenüber (173). Dabei kann die Einführung von Dreiklangstypen im Zwischenbereich zwischen konsonant und dissonant "als der eigentliche Ursprung einer (deutschen) Stufentheorie mit einer voll integrierten 7 . Stufe gelten« (170).

13 Stölzel, Practischer Beweiß, § 4ff.

14 Vgl. Vogt 2015, 116. 


\section{INHALT}

Stölzels Anleitung gliedert sich in Proportionslehre (10 Kapitel), Moduslehre (3), Klausellehre (1), Intervalllehre (5), Trias-harmonica-Lehre (1), Fux'sche Hauptregeln (1), Kontrapunktlehre (Gattungen) (7), Rezitativlehre (1) und Imitationslehre (5) (45). Vogt geht im Kommentar nicht auf alle Inhalte gleichermaßen ein, sondern fokussiert diejenigen, die die konzeptionellen Neuerungen und damit den Stellenwert von Stölzels Traktat begründen. Dies ist bereits im Aufbau der Anleitung angelegt, die derjenigen von Fux' Gradus ähnelt, aber an entscheidenden Stellen Kapitel zu zeitgenössischen Ausprägungen als direkte Gegenüberstellung zum alten Stil enthält: So folgt u. a. dem XXIII. Kapitel "Vom Contrapuncto simplici« (258) eines »De Contrapuncto simplici moderno" (268). Der zentrale Teil des Kommentars widmet sich entsprechend den vier Themen Intervalllehre, Tonartenlehre, Dreiklangslehre sowie Contrapunctus-simplex-Lehre. Vorangestellt ist eine Aufarbeitung der Stölzel-Rezeption, die genaue Beschreibung der einzigen bekannten Quelle aus der Sammlung Poelchau und die Einordnung in Stölzels Schriften insgesamt ${ }^{15}$ sowie eine Übersicht über Aufbau und Inhalt im Vergleich zu Fux.

Mit Rückblick auf Stölzels Vorläufer von den Griechen bis zu Zarlino, Galilei, Lippius und Baryphonus analysiert Vogt, wie Stölzel die Intervalle nicht nur nach Einfachheit der Proportion, sondern auch in Bezug auf die strias harmonicas kategorisiert (53f.); dabei arbeitet er auch die Widersprüche in der teils inkonsistenten Argumentation Stölzels zur Klassifizierung von Konsonanzen heraus (60). Auf eine Analyse der »von zugrunde liegenden Trias-Strukturen« (62) geprägten Perspektive Stölzels auf Modi und Klauseln folgt eine Dis-

15 Neben dem Practischen Beweiß (Druck und Abschriften) und der Anleitung sind eine $» \mathrm{Ab}$ handlung über die Composition « (Konvolut aus Rezitativlehre und einer Satzlehre) sowie zwei weitere zusammengebundene Traktate (»Kurzer und gründlicher Unterricht« (s.u.), und »Von dem ordentlichen und natürlichen Gebrauch der Dissonanzen in Ligatura «) als Manuskript erhalten. Der Rezitativtraktat liegt in Edition und Kommentar von Werner Steger vor (Steger 1962). kussion der neuen Tonarten Dur und Moll nebst Ausweichungstönen, wobei in Moll alle Dreiklänge als »leitereigen« inbegriffen sind, die sich aus dem Ambitu modorum mit den doppelten Stufen 6 und 7 ableiten lassen (82ff.). Viel Raum nimmt aufgrund ihres Innovationspotenzials die Dreiklangslehre ein, insbesondere die Emanzipation der »sonderlich heut zu Tage gebräuchlichen Harmonien", wozu nicht nur verminderter und übermäßiger Dreiklang zählen: »[...] es findet sich auch wohl noch eine deficiens, welche aus einer Quinte deficiente und Tertia deficienti zugleich bestehet, [...] [und] man trift auch Syzygias an, die auf eine Triade deficienti beruhen, so aus der Quinte deficienti und tertia majori bestehet $[\ldots] \ll$ (247). ${ }^{16}$ In das Kapitel eingeschoben ist ein Exkurs zur Vorgeschichte der Kategorisierung von vermindertem und übermäßigem Dreiklang in der Trias-harmonica-Lehre des 17. Jahrhunderts. Neben der Integration eines modernen Tonartenverständnisses und der "Rechtfertigung" der oben genannten neuen Klangtypen im modernen Contrapunctus simplex (156ff.) ist die satztechnische Herangehensweise Stölzels bezeichnend, der - anders als beim "alten« Contrapunctus simplex - den modernen zweistimmigen Contrapunctus simplex direkt als Außenstimmensatz konzipiert (121). Dieser Abschnitt wird ergänzt durch einen Exkurs zu Stölzels Choralharmonisierungslehre (s.u.).

In einem stringent zusammenfassenden Kapitel benennt Vogt schließlich »Traditionsbewusstsein und historische Kontinuität« (165), »Innovation " (170) und "Kompilation« (171) als die wesentlichen Merkmale der in der Anleitung ausgeprägten Stölzel'schen Musiktheorie.

16 Stölzel geht an dieser Stelle nicht auf typische Formen (wie etwa im Falle des doppelt verminderten Dreiklangs auf die Verwendung als übermäßiger Sextakkord) ein. Er bringt aber später im Kapitel zum »modernen« Contrapunctus simplex ein vierstimmiges Beispiel mit übermäßigem Sextakkord und kommentiert: „Obwohl man ehemals folgenden Satz für unzuläßig gehalten, so wird er doch heut zu Tage öfters gebraucht, und bestehet darinnen daß seine Basis der Medius Triadis deficientis cum Tertia deficienti ist, als wovon Cap. XX. §. 8 gesagt worden« (271f.). 


\section{BEMERKENSWERTE ASPEKTE IN EDITION UND KOMMENTAR}

\section{Stölzels Traktat in der kommentierten Edition}

Stölzel orientiert sich viel stärker als Fux an der (historischen und gegenwärtigen) Praxis. Es bedarf weniger der Begründung durch axiomatische Grundsätze, vielmehr zählt, was in Kompositionen auftritt und "gut klingt«. ${ }^{17} \mathrm{Er}$ verlässt sich aber nicht allein auf Gewohnheit und Geschmack, sondern bemüht sich um einen modernen Theorie-Anspruch, der sich nicht mit dem Nebeneinander von $>$ musica theorica und smusica practica` zufrieden gibt, sondern das eine aus dem anderen zu begründen sucht. Gerade Stölzels Versuche der Analogiebildung zwischen alten und neuen Prinzipien wirken jedoch zuweilen etwas bemüht. So bestehe etwa die "Verwandtschaft « zwischen den alten und modernen "Modis" darin, dass beider Charakteristika jeweils auf die harmonische und arithmetische Teilung zurückzuführen seien: Die alten Modi unterscheiden sich durch die jeweilige Teilung der Oktave (authentisch vs. plagal), die neuen aber durch die der Quinte (Dur vs. Moll) (216f.). Auch sonst ist seine Argumentation mitunter gewagt: So untersucht er Beispiele, die für ihn zur Gattung Contrapunctus simplex zählen, und leitet daraus ab, dass die dort auftretenden Klänge als konsonant zu betrachten seien (158). Folglich kann sogar der übermäßige Sextakkord als Konsonanz gebraucht werden, wodurch wiederum die trias deficiens cum tertia deficienti aufgewertet wird (160).

Seine enge Anknüpfung an die kompositorische Praxis zeigt sich sowohl in raktuellen Abschnitten wie dem Rezitativ-Kapitel als auch in der Ähnlichkeit seiner Lehrbeispiele zu eigenen Kompositionen. Obwohl Stölzel gelegentlich Vorgehensweisen detailliert erklärt - etwa im Kapitel "Vom Recitativ« (308ff.), in dem systematisch metrisch-rhythmische Gestaltung,

17 Im Zusammenhang mit Sorges Intervallbegriff beschreibt auch Holtmeier diese neue Dominanz der Hörerfahrung und die zu Beginn des 18. Jahrhunderts in Italien und Deutschland aus ihr entspringende sensualistische Kritik an tradierten musiktheoretischen Kategorien. Vgl. Holtmeier 2017, 191.
Bewegungsrichtung und Klauselbildung in Abhängigkeit von Textbetonung und Interpunktion erläutert und demonstriert werden - handelt es sich insgesamt um einen sehr wenig redseligen, knappen und kompakten Traktat. Häufig sind es gelungene Beispiele ${ }^{18}$, die Sachverhalte oder satztechnische Entscheidungen verdeutlichen, sodass der Text mitunter auf ein eher nachahmendes als abstrahierendes Lernen zielt. ${ }^{19}$ Angaben, auf welche Schriften oder Theoretiker Stölzel sich bezieht, fehlen, sodass (referiertes) Tradiertes und (eigenes) Neues nicht auf den ersten Blick voneinander zu unterscheiden sind. Sehr hilfreich sind daher Vogts Anmerkungen, die zum einen offen legen, wo Stölzel Fux' Beispiele übernimmt oder variiert ${ }^{20}$ und wo er sich auf andere Autoren bezieht, zum anderen Schreibfehler und unvollständige Erläuterungen aufklären und auch Anhaltspunkte für mögliche Vorbilder und Prinzipien hinter den Beispielen geben.

18 Eine besonders schöne Sammlung solcher Beispiele findet sich im Kapitel "Von den dreystimmigen Fugen« (334ff.). Hier erweitert Stölzel die knappen Beispiele zur »erdichteten Clausul « in der Fux'schen Vorlage (Fux/Mizler 1742, 131f., Tab. XXIVf.) auf fünf Paragraphen zur Kadenzflucht: „Hier kann man auch die Cadenzen zierlich ausfliehen, und also wird nöthig seyn, von dieser Sache [...] etwas weitläuftiger zu reden« (ebd., 335). Es folgen zahlreiche vierstimmige(!) Beispiele für Modulationen, Ausweichungen sowie zwischendominantische und trugschlüssige Wendungen, bevor das erste Exempel zur dreistimmigen Fuge unvermittelt zum Fux'schen Vorbild zurückkehrt (ebd., 341).

19 So bringt Stölzel etwa zur »Brechung» der gebundenen Dissonanzen im ,Contrapunctus floridus` (298) eine Sammlung typischer Auszierungsfloskeln, ohne jedoch darauf hinzuweisen, dass sie in der hier vorgestellten sequenzierenden Anlage stilistisch eher dem Barock als dem 16. Jahrhundert zuzuordnen sind.

20 Stölzel überträgt im dreistimmigen Contrapunctus simplex die Fux'schen Beispiele ohne jegliche Änderung; beim vierstimmigen Contrapunctus simplex hingegen wird lediglich der Cantus firmus übernommen, die anderen drei Stimmen aber neu hinzugefügt. 


\section{Kommentar}

Da der Kommentar sich auf die "Modernisierungs-Felder « konzentriert, werden andere Teile (wie etwa Kap. XXVI »De Contrapuncto ligato «) zwar innerhalb der Edition kommentiert, aber kaum in einen größeren Zusammenhang eingeordnet, sodass Verknüpfung und Vergleich etwa mit zeitgenössischen Generalbasslehren von den Leser*innen selbst geleistet werden müssen. Sehr nützlich sind dafür die Aufschlüsselungen verwandter Begriffe, wie etwa der fast bedeutungsgleichen Termini 'Syzygia', 'Conjunctios, 'Concentus hältnis zum Begriff Akkord (68f). Eine große Bereicherung stellen die Exkurse zur Triasharmonica-Lehre des 17. Jahrhunderts und zu Stölzels (Choral-)Harmonisierungslehre aus dem Manuskript »Kurzer und gründlicher Unterricht $[\ldots] \ll^{21}$ dar $(36,126)$. Letztere ergänzt den eigentlich essentiellen, am wenigsten mechanischen Teils der Harmonisierung, wie man nämlich überhaupt »ordentlicher u. natürlicher weiße« (134) zu einem einfachen, wohlklingenden und harmonisch gedachten Außenstimmensatz kommt. Die Veranschaulichung Stölzel'scher Prinzipien durch die Analyse eines Chorals aus der Brockes-Passion ${ }^{22}$ zeigt zugleich, dass die empfohlenen Arbeitsschritte nicht als lediglich propädeutische Übungen zu verstehen sind, sondern zu ästhetisch vollgültigen Kompositionen führen können (126ff.). Ebenso erhellend sind detaillierte Untersuchungen einzelner Beispiele, z. B. des nur zweistimmig ausgeführten "General Bass Exempe/« zur Veranschaulichung der gebundenen Dissonanzen (289). Drei Vorschläge Vogts zur drei- und vierstimmigen Aussetzung zeigen, wie Stölzel durch Mehrdeutig- keiten in der Bezifferung zu einer kreativen Konzeption der Mittelstimmen anregt.

\section{ZUSAMMENFASSUNG}

Vogt hat durch seine sorgfältige editorische Arbeit an Stölzels Traktat ein durchweg überzeugendes Buch vorgelegt. Im bestens strukturierten Kommentar arbeitet er plastisch die wesentlichen Neuerungen und Eigenheiten von Stölzels Theorie einschließlich der zugehörigen Begrifflichkeit heraus. Mitunter könnte der Kommentar etwas kompakter sein, einzelne Punkte wirken überausführlich erklärt und manche Wiederholungen und Abbildungen wären durchaus entbehrlich. Andererseits soll der Text ja auch Leser*innen dienen, die lediglich eine Kontextualisierung einzelner Passagen des Traktats suchen. Die gelegentlich auftretenden Redundanzen haben also ihre Begründung in der Zielsetzung des Buches. Ein umfangreicher Apparat von Anmerkungen und Verweisen bindet die Untersuchung in den aktuellen Kontext der Forschung zur barocken Musiktheorie ein und eröffnet so - bei aller Stringenz und Fokussierung auf den Traktat und seinen unmittelbaren Kontext - eine weite Perspektive. Vogts treffender und flüssiger Schreibstil, die klare Verständlichkeit und nicht zuletzt ein gutes Lektorat machen das Buch angenehm zu lesen.

\section{Almut Gatz}

21 Gottfried Heinrich Stölzel (o.D.), Kurzer und gründlicher Unterricht, wie ein Liebhaber der Music, welcher die Intervalla Musica kennet, und durch die Noten aufzuschreiben weiß, in einer kurzen Zeit, einen Contrapunctum Simplicem, doch ohne Sexten, mit vier Stimmen zu sezen erlernen kan, Staatsbibliothek zu Berlin, Signatur: Mus.ms.theor. 839, f. 1-16v.

22 Die Harmonisierung der ersten Hälfte entspricht genau der im Lehrbuch vorgestellten, viel freier ist dagegen die zweite: mehr Sextakkorde, überraschende Nebenstufen, weniger eng am Sopran geführte Mittelstimmen (154). 


\section{Literatur}

Fux, Johann Joseph (1742), Gradus ad Parnassum oder Anführung zur regelmässigen musicalischen Composition, übers. von Lorenz Christoph Mizler von Kolof, Leipzig: Mizlerscher Bücherverlag.

Heffter, Moritz (i.V.), Die Plejades Musicæ des Henricus Baryphonus. Edition und Kommentar, Hildesheim: Olms.

Holtmeier, Ludwig (2017), Rameaus langer Schatten. Studien zur deutschen Musiktheorie des 18. Jahrhunderts, Hildesheim: Olms.

Printz, Wolfgang Caspar (1696), Phrynis Mitilenæus, oder Satyrischer Componist, Dresden: Miedt und Zimmermann.

Sprick, Jan Philipp (2009), „Die strias harmonicas in der deutschen Musiktheorie des 17. Jahrhunderts. Zwischen Theologie und musikalischer Praxis», in: Musik an ihren Grenzen: Neue und Alte Musik, hg. von Angelika Moths, Markus Jans, John MacKeown und Balz Trümpy, Bern: Lang, 369391.
Steger, Werner (1962), G. H. Stölzels »Abhandlung vom Recitativ« [Edition und Kommentar], Diss., Universität Heidelberg.

Stölzel, Gottfried Heinrich (1725), Practischer Beweiß, wie aus einem nach dem wahren Fundamente solcher Noten-Künsteleyen gesetzten Canone perpetuo in hypo dia pente quatuor vocum, viel und mancherley, Theils an Melodie, Theils auch nur an Harmonie, unterschiedene Canones perpetui à 4 zu machen seyn. Der Warheit, und einigen MusicFreunden zu gefallen, dem Druck überlassen, von G. H. S., o.O.

Vogt, Florian (2015), „Gottfried Heinrich Stölzels Fux-Rezeption. Zur Rolle des salten Kontrapunkts und der strias harmonicas in der Kompositionsausbildung zu Beginn des 18. Jahrhunderts«, in: Musiktheorie und Komposition. XII. Jahreskongress der Gesellschaft für Musiktheorie Essen 2012, hg. von Markus Roth und Matthias Schlothfeldt, Hildesheim: Olms, 115-126.

Gatz, Almut (2019): Florian Vogt, Die »Anleitung zur musikalischen Setzkunst« von Gottfried Heinrich Stölzel (1690-1749). Edition und Kommentar, Neumünster. von Bockel 2018. ZGMTH 16/1, 137-142.

https://doi.org/10.31751/1004

(C) 2019 Almut Gatz (almut.gatz@hfm-wuerzburg.de)

Hochschule für Musik Würzburg

Dieser Text erscheint im Open Access und ist lizenziert unter einer

Creative Commons Namensnennung 4.0 International Lizenz.

This is an open access article licensed under a

(c)

Creative Commons Attribution 4.0 International License.

eingereicht / submitted: 16/04/2019

angenommen / accepted: 11/05/2019

veröffentlicht / first published: 30/06/2019

zuletzt geändert / last updated: 30/06/2019 